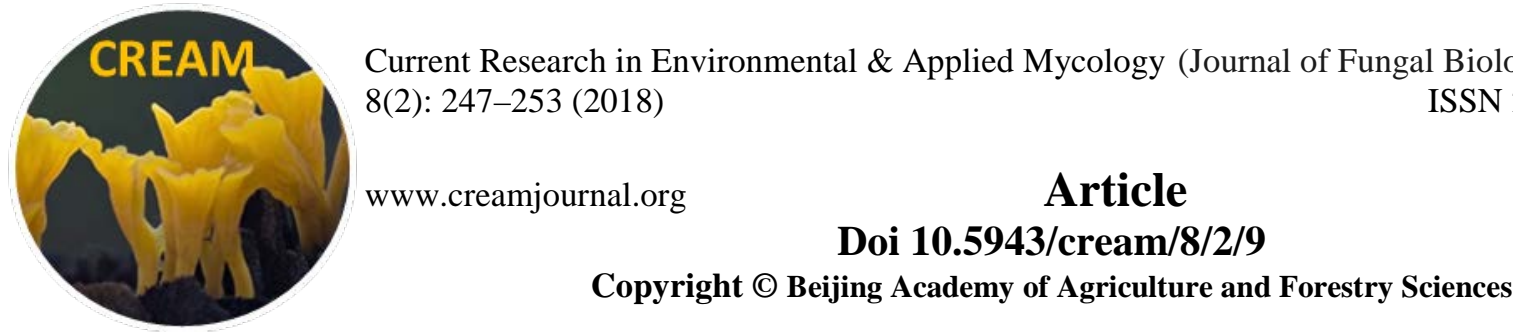

\title{
Macrolepiota aberdarense, a new edible mushroom from Kenya
}

\section{Mbaluto $C^{1}$, Runo $\mathrm{S}^{1}$, Wanyoike $\mathrm{W}^{3}$, Onyango $\mathrm{C}^{2}$, Kimani $\mathrm{W}^{4}$, Jagger $\mathbf{H}^{4}$ and Otieno DO ${ }^{2}$}

${ }^{1}$ Department of Biochemistry and Biotechnology, Kenyatta University, P.O BOX 43844-00100 Nairobi, Kenya.

${ }^{2}$ Food Technology Division, Kenya Industrial Research and Development Institute, P.O BOX 30650-00100 Nairobi, Kenya

${ }^{3}$ Department of Botany, Jomo Kenyatta University of Agriculture and Technology, P.O BOX 62000-00200 Nairobi, Kenya

${ }^{4}$ Biosciences eastern and central Africa, International Livestock Research Institute, P.O BOX 30709-00100 Nairobi, Kenya

Mbaluto C, Runo S, Wanyoike W, Onyango C, Kimani W, Jagger H, Otieno DO 2018 Macrolepiota aberdarense, a new edible mushroom from Kenya. Current Research in Environmental \& Applied Mycology (Journal of Fungal Biology) 8(2), 247-253, Doi $10.5943 /$ cream/8/2/9

\begin{abstract}
A new Macrolepiota species from the Aberdare Forest in Kenya is described and illustrated. The larger basidiomata with yellow brownish to brownish granular squamules, distinct umbo, larger basidia and smaller ellipsoid basidiospores form remarkable features that separate this species from previously published members belonging to the genus Macrolepiota. The phylogenetic analyses based on ITS-rDNA sequences further supported this distinction.
\end{abstract}

Key words - Agarics - ITS rDNA - morphology - phylogeny - taxonomy

\section{Introduction}

The genus Macrolepiota Singer contains many species of edible mushrooms (Ge et al.2010). It is composed of Basidiomycetes fungi belonging to the class Agaricomycetes and order Agaricales (Singer 1948). Traditionally, Macrolepiota species have been identified on the basis of their morphology, particularly basidiomata structures including squamules, lamellae, partial veils in the form of annulus, clamp connection on the septa of the hyphae in lamellae and basidiospores (Singer 1948, Guzmán et al. 1993, Vellinga \& Yang 2003). However, use of morphological characters proved to be unreliable and when used solely can lead to many open questions and controversies. Modern fungal systematics via incorporation of molecular markers have been reported therefore to provide finer-scale resolution of phylogenetic relationships (Taylor \& McCormick 2008, Ge et al. 2010). The most widely used molecular marker for discrimination of fungal species is the internal transcribed spacer (ITS) rDNA region (Chillali et al. 1998, Koljalg et al. 2001, Horton 2002). Similarly, the ITS markers have provided reliable information on geographic speciation among many fungal lineages including mushrooms (Zervakis 2004). Currently, over 100 Macrolepiota species have been described worldwide and are listed in www.indexfungorum.com (Kirk et al. 2008). The most described Macrolepiota species are of Europe, Asia, Australia and North America origin (Ge et al. 2010). Only a few species of Macrolepiota have been reported from Africa (Hussein et al. 2014, Pegler \& Rayner 1969). 
Information on Macrolepiota species in Kenya is quite limited. The distribution of this species is limited to certain parts of the country and so does the consumption. Macrolepiota species provides major part of nutrition among indigenous communities living around the Aberdare Forest. Fresh mushrooms are collected and cooked and the surplus is dried in the sun for future use. Macrolepiota species is also used for medicinal purposes among the local communities. Interest in commercial production of Macrolepiota in other parts of Kenya is therefore increasing, not only to provide nutrition, but also culinary options such as those provided by other edible mushroom species such as Agaricus L., Pleurotus (Fr.) P. Kumm., Volvariella Speg. and Lentinula Earle that are currently under commercial production. However, man-made and natural calamities including deforestation, frequent fire out breaks and prolonged droughts have threatened the loss of gene pool of forest biodiversity including edible mushrooms. In this study, we applied morphological and molecular evidence to infer taxonomic status of Macrolepiota species collected from the Aberdare Forest.

\section{Materials \& Methods}

\section{Sample collection}

Sampling of Macrolepiota species was done in the Aberdare Forest in central Kenya. The forest is situated at an altitude of $1829 \mathrm{~m}-4001 \mathrm{~m}$ above the sea level and covers an area of 980 $\mathrm{km}^{2}$ (KFS 2009). The forest vegetation comprises of Lobelia deckenii subsp. sattimae, Helichrysum gloria-dei and Alchemilla hageniae as the most endemic plant species (KFS 2012). Fruiting bodies of Macrolepiota sp. were carefully dug from the ground below their stipe with a knife. Photos were taken of fresh collections. The exact localities of the isolates were recorded using GPS machine (Garmin Inc., Kansas, USA). Culture specimens have been deposited at the KIRDI mushroom culture collection centre under the accession numbers KMC513-1 to KMC513-8.

\section{Morphological examination}

Macromorphological features of the fresh basidiomata were examined based on field notes and photos. Microscopic observations were performed on fresh and dried material with a compound light microscope (Reid 1984). Specific attention was given to structures of the basidiospores, basidia, cystidia, pileus hyphae and stipe hyphae. Sections of basidiocarp were cut and stained with aqueous solution of $\mathrm{KOH}$, Cotton blue, 1\% ammoniacal Congo red or Melzer's reagent. Microscopic structures including basidia, cheilocystidia, cystidia and squamules were observed in 5-10\% KOH and with Congo-red then drawn using free hand. Photos of basidiocarp were taken.

The abbreviation $[\mathrm{n} / \mathrm{m} / \mathrm{p}]$ denotes $\mathrm{n}$ basidiospores measured from $\mathrm{m}$ basidioma of $\mathrm{p}$ collections in $5-10 \% \mathrm{KOH}$ solution. A minimum of 20 individual basidiospores were measured from each basidioma using a calibrated ocular micrometer at $100 \times$ objective lens. Dimensions for basidiospores are recorded as (a-) b-c (-d). The range b-c includes a minimum of $90 \%$ of the taken values. The values ( $\mathrm{a}-$ and $-\mathrm{d}$ ) which were considered as extreme are enclosed in parentheses. Q represents "length/width ratio" of a spore in side view; $\mathrm{Q}_{m}$ represents mean $\mathrm{Q}$ of all basidiospores \pm sample standard deviation.

\section{Molecular analysis}

Genomic DNA was extracted from dried pileus tissue using procedure described previously (Otieno et al. 2015) with some modifications. The internal transcribed spacer (ITS1+5.8S+ITS2) region of the ribosomal DNA was amplified separately with ITS5 (forward) and ITS4 (reverse) primers (White et al. 1990). PCR products were treated with ExoSAP-IT (USB Corporations, Cleveland, OH, USA), then sequenced with a Big dye terminator cycle sequencing kit (Applied Biosystems, Foster City, California, USA) following the manufacturer's protocol. Sequencing was done in an Applied Biosystems 377 XL automated DNA sequencer (Applied Biosystem, Foster City, USA). Sequences were aligned with MAFFT 7.0 (Katoh \& Standley 2013). jModelTest 2.1.9 (Darriba et al. 2012) was used to estimate the best-fit nucleotide substitution model. 
Phylogenetic reconstructions were carried out using RAxML 8.2.4 (Stamatakis 2014) and Mr Bayes 3.2.6 (Ronquist et al. 2012). Maximum-likelihood analysis was conducted using the "autoMR" option of automatic 'bootstopping' (Pattengale et al. 2010) under the GTR+CAT nucleotide substitution model. The Markov chain Monte Carlo was run for 5 million generations under the HKY $+\mathrm{G}$ nucleotide substitution model. Each tree shows the topology obtained with the maximum-likelihood method; ML bootstrap support values $\geq 75 \%$ are shown and branches with Bayesian posterior probabilities $\geq 0.90$ are thickened. Leucoagaricus barssii (Zeller) Vellinga and Leucoagaricus meleagris (Gray) Singer were used in the phylogenetic analyses as outgroup taxa. Additional published ITS sequences from Macrolepiota species were included in the alignment. Nucleotide sequence of M.aberdarense was submitted to National Center for Biotechnology Information (NCBI) database under GenBank accession numbers KP97461-KP974618.

\section{Results}

\section{Phylogenetic analyses}

The phylogram based on ITS rDNA sequences revealed four distinct clades of the Aberdare isolates. The Aberdare strains were well separated from their GenBank homologs with 100\% bootstrap support and 1posterior probability values. Maximum likelihood (ML) and Bayesian algorithm analyses of aligned ITS rDNA sequence data revealed similar tree topologies with some differences in bootstrap and posterior probability values (Fig. 1). Initial nucleotide comparisons with Basic Local Alignment Search Tool (BLAST) against the National Center for Biotechnology Information (NCBI), USA GenBank database revealed over 98\% sequence similarity with Macrolepiota dolichaula.

\section{Taxonomy}

Macrolepiota aberdarense Mbaluto \& Otieno, sp. nov. Figs 2, 3

Basionym: Macrolepiota dolichaula Fr., (Berk). Pegler \& Rayner

= Agaricus dolichaulus Berk. \& Br. in Trans. Linn. Soc. 27: 150 (1870).

= Lepiota dolichaula (Berk. \& Br.) Sacc., Syll. Fung. 5: 32 (1887)

Heterotypic synonyms: refer to Pegler $(1977,1983)$ and Ge et al 2014.

Holotype - Mathioya, Nyeri County, coll. by O.D. Otieno

Mycobank: MB823875

Etymology: name referring to the location where isolate was obtained

\section{Diagnosis}

Pileus 9-22 cm diam., 0.3-0.4 cm thick, campanulate to plano-convex, broadly umbonate toward the centre; yellow brown to brownish squamules; margins incurved, appendiculate and sometimes inconspicuously short striated. Hymenophore lamellate, lamellulae intercalated when young, short, highly to moderately crowded, bulging and free and sometime adnexed, margin colour brownish whitish and creamish. Stipe 5-21 $\times 0.8-2.0 \mathrm{~cm}$, white when young to whitish when mature; subcylindrical, slightly tapering toward apex, hollow with tiny farinose granules, slightly bulbous at the base. Annulus ascending, simple and whitish, slightly membranous. Context whitish. Taste mild.

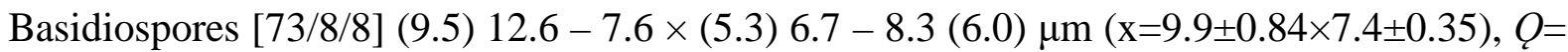
(1.42) $15.1 \times 7.5, Q_{m}=1.9-2.2$; side view ovoid to ellipsoid, front view ellipsoid; thick-walled (about $0.7 \mu \mathrm{m})$, smooth, congophilous and inamyloid in Congo red and Melzer's reagent respectively, germ pore caused by an interruption in the episporium on the rounded apex; Basidia 26-34×11-15 $\mu \mathrm{m}$, clavate, thin-walled, hyaline, 4-spored, four short sterigmata up to $4.5 \mu \mathrm{m}$ long; Cheilocystidia 21-30×12-15 $\mu \mathrm{m}$, thin-walled, hyaline, clavate to broadly clavate. Pleurocystdia absent. Squamules on pilei palisades of short, frequently branched, subcylindric, clampless hyphae with terminal elements, subcylindric to subfusiform, 8-16 $\mu \mathrm{m}$ in diam., hyaline or with yellowish vacuolar 


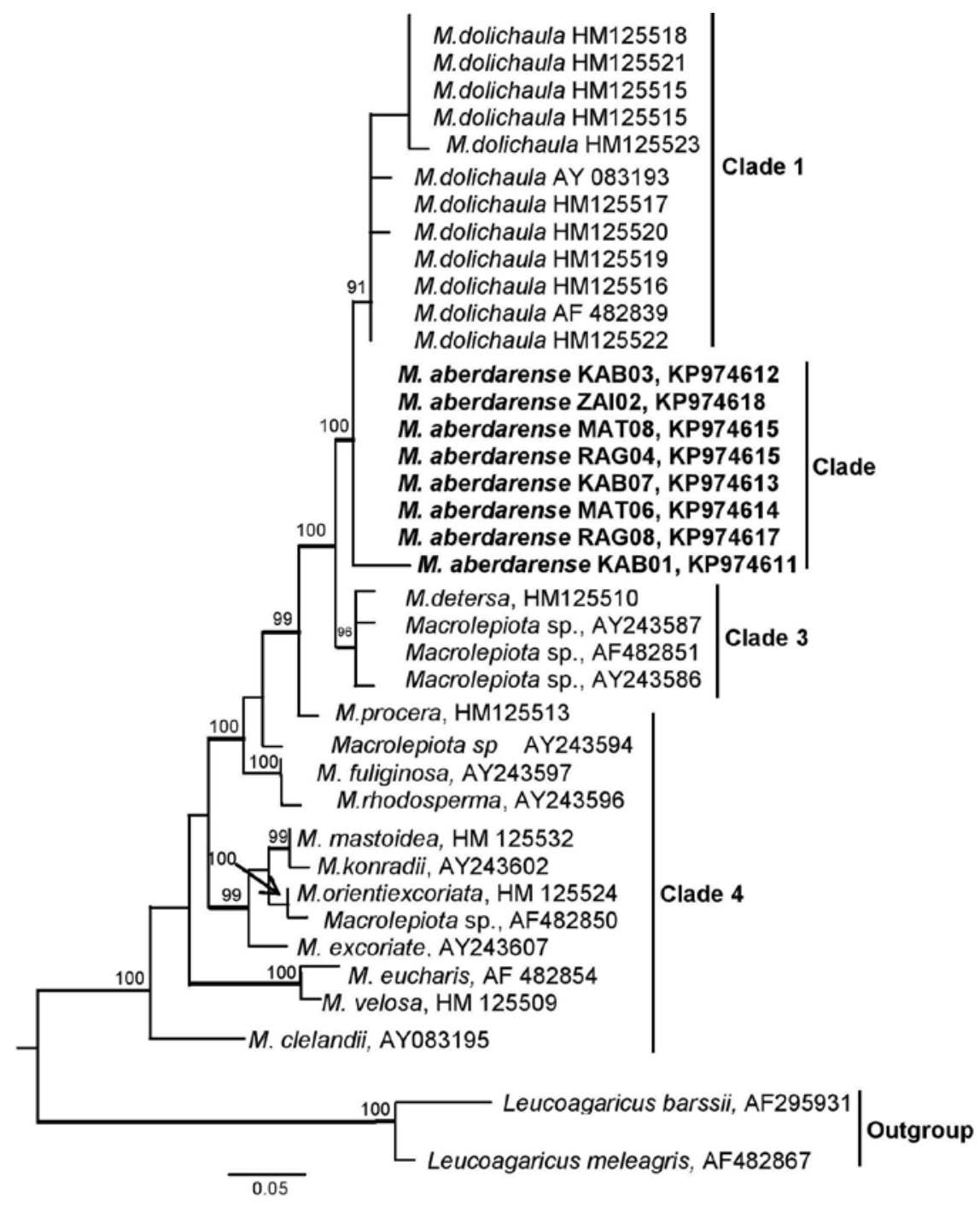

Fig. 1 - Phylogenetic placement of the new species within the genus Macrolepiota based on ITS sequences analysis. Maximum-likelihood bootstrap support values $\geq 70 \%$ are shown; branches with Bayesian posterior probabilities $\geq 0.90$ are thickened.

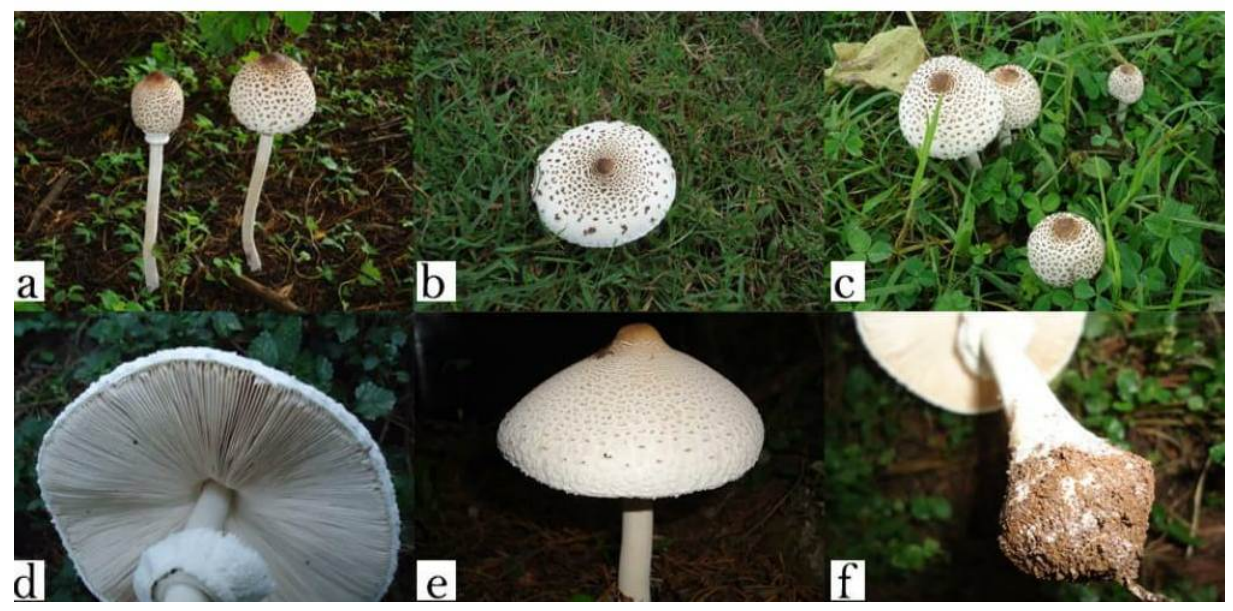

Fig. 2 - Basidiocarp of Macrolepiota aberdarense a long stipe, basidiocarp campanulate when young and become convex to plano-convex with age. $\mathrm{b}$ pileus covered with yellow brownish to brownish granular squamules. c solitary to scattered occurrence in nature. d reverse side of basidiocarp showing white lamellae and annulus. e Pileus with acute umbo at disc (holotype MAT08) f Volviform marginate bulb at the base of stipe. Scale bars=10 $\mathrm{mm}$. 


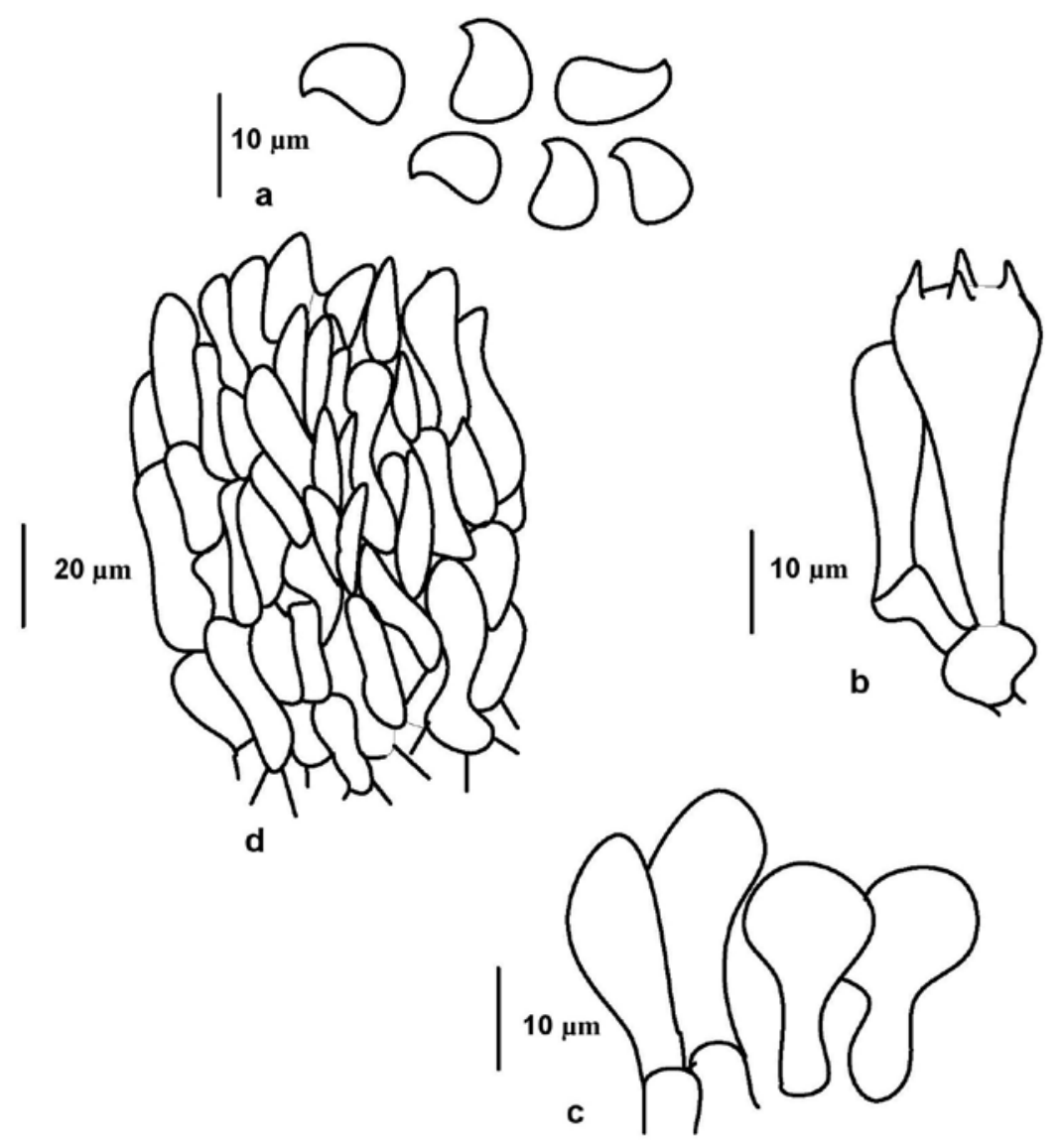

Fig. 3 - Micromorphological characters of Macrolepiota aberdarense (Holotype MAT 08). a Basidiospores. b Basidia. c Cheilocystidia. e Squamules.

pigment, thin-walled to slightly thick-walled. Clamp connections present at the base of basidia and cheilocystidia.

Habitat and known distribution - Solitary or scattered with preference to open grassland particularly along the footpaths.

Materials examined - KENYA. Nyeri County, Aberdare National Park, Mathioya Forest Reserve, S0²3'34.77", S36 48'50.55", elev. 2214 m, 8 April 2012, coll. by O.D. Otieno, MAT08 (KP974615).

\section{Key to the taxa of Macrolepiota from Kenya}

1. Basidiomata medium-sized to large (9-22 cm diam), pileus yellow brown to brownish, lamellae distant, stipe subcylindrical with white to whitish surface, basidiospore (12.6-17.6×6.7- 8.3) $\mu \mathrm{m}$, cheilocystidia thin-walled, hyaline, clavate to broadly clavate, narrow distribution

M. aberdarense

1.' Basidiomata small to small-to medium-sized, pileus trichodermal palisade, lamellae crowded, stipe cylindrical with white to 'Pale Ochraceous-Buff' surface, 10.5-I4.5 x 7-10 (12.5 x 8.5) $\mu \mathrm{m}$, cheilocystidia clavate with rounded apex, hyaline, thin-walled, narrow distribution

M. dolichaula

\section{Discussions}

Macrolepiota aberdarense is characterized by larger basidiocarp, average pileus size 9-22 $\mathrm{cm}$ diam, 3-4mm thick, campanulate to plano-convex, broadly and acutely umbonate toward the centre; yellow brown to brownish squamules; margins incurved, appendiculate and sometimes 
inconspicuously short striate. Hymenophore lamellate, lamellulae intercalated when young, short, highly to moderately crowded, bulging and free and sometime adnexed, margin colour brownish whitish and creamish. Stipe white when young to whitish when mature; subcylindrical, 5-21×0.8$2.0 \mathrm{~cm}$, slightly tapering toward apex, hollow with tiny farinose granules, slightly bulbous at the base. Annulus ascending, simple and whitish, slightly membranous. Context whitish. Taste mild.

Basidiospores (12.6-17.6×6.7- 8.3) $\mu \mathrm{m}$; cheilocystidia 21-30 × 12-15 $\mu \mathrm{m}$, narrowly clavate to clavate; basidia 26-34 $\times 11-15 \mu \mathrm{m}$; sterigmata up to $4.8 \mu \mathrm{m}$ long. In comparison, previously described M. dolichaula (Pegler \& Rayner 1969, Ge et al. 2010) which is closest sister of $M$. aberdarense hardly exceeded $20 \mathrm{~cm}$, cheilocystidia 20-33 × 11-15 $\mu \mathrm{m}$, clavate to broadly clavate; basidia 28-33 $\times 11-15 \mu \mathrm{m}$, sterigmata up to $4.5 \mu \mathrm{m}$ long. Most variations were within the range generally reported for Macrolepiota dolichaula Fr., (Berk). Pegler \& Rayner (Singer 1948, Pegler \& Rayner 1969).

Based on the phylogenetic analyses, we found no evidence for the distribution of $M$. aberdarense across continents; thus, this species may be endemic to Africa. Similarly, there was no sequence diversity detected within our collections, possibly due to small sample size used. Collections from a wider geographic range could reveal more Macrolepiota diversity in Kenya, as some species i.e. M. procera and M. dolichaula have been reported in Tanzania and DRC Congo, (Pegler \& Rayner 1969, Hussein et al. 2014), a few hundred kilometers from the Aberdare Forest. Given the high sequence similarity between these two speices, we hypothesize there was recent split or lack of gene flow between these two species. Similar changes in gene patterns have been reported in natural population of fungi (Hiremath et al. 2008, Xu et al. 2008). However, since morphological, molecular and biogeography data clearly distinguish the Aberdare strains from previously described Macrolepiota species; we therefore described the Aberdare strains as a new species within the genus Macrolepiota.

\section{Acknowledgements}

This study was financially supported by the Australian government through the African Biosciences Challenge Fund. The authors are grateful to the Kenya Forest Service for providing permission to access mushroom genetic resources from the Aberdare Forest. The authors sincerely appreciate inputs from Prof. Gregory Bonito and Dr. Alessandro Desirò (Michigan State University, MI, USA).

\section{References}

Chillali M, Wipf D, Guillaumin JJ, Mohammed C, Botton B. 1998 - Delineation of the European Armillaria species based on the sequences of the internal transcribed spacer (ITS) of ribosomal DNA. New Phytology 138:553-561.

Darriba D, Taboada GL, Doallo R, Posada D. 2012 - jModelTest 2: more models, new heuristics and parallel computing. Nature Methods 9:772.

Ge ZW, Zhu LY, Vellinga EC. 2010 - The genus Macrolepiota (Agaricaceae, Basidiomycota) in China. Fungal Diversity 45:81-98.

Guzmán G, Bandala VM, Allen JW - 1993. A new bluing Psilocybe from Thailand. Mycotaxon 46:155-160.

Hiremath SS, Chowdhary A, Kowshik T, Randhawa HS et al. 2008 - Long-distance dispersal and recombination in environmental populations of Cryptococcus neoformans var. grubii from India. Microbiology 154:1513-1524.

Horton TR. 2002 - Molecular approaches to ectomycorrhizal diversity studies: variation in ITS at a local scale. Plant Soil 244:29-39.

Hussein JM, Tibuhwa DD, Mshandete AM, Kivaisi AK. 2014 - Molecular phylogeny of saprophytic wild edible mushroom species from Tanzania based on ITS and nLSU rDNA sequences. Current Research in Environmental \&amp; Applied Mycology 4:250-260. 
Katoh K, Standley DM. 2013 - MAFFT multiple sequence alignment software version 7: improvements in performance and usability. Molecular Biology and Evolution 30:772-780.

KFS. 2009 - Kenya Forest Service Strategic Plan 2009/10 - 2013/14. KFS, Nairobi. 2-94

KFS. 2012 - Aberdare Forest Reserve Management Plan, in: Environment (Ed.), Kenya Forest Service, Nairobi.

Kirk PM, Cannon PF, Minter DW, Stalpers JA. 2008 - Dictionary of the fungi. 10th ed. CABI, Wallingford.

Koljalg U, Jakucs E, Bo'ka K, Agerer R. 2001 - Three ectomycorrhizae with cystidia formed by different Tomentella species as revealed by rDNA ITS sequences and anatomical characteristics. Folia Cryptogamica Estonica 38:27-29.

Otieno DO, Onguso J, Matasyoh LG, Onyango C et al. 2015 - Genetic diversity of the Kenyan oyster mushroom (Pleurotus). Mycologia 107:32-38.

Pattengale ND, Alipour M, Bininda-Emonds OR, Moret BM, Stamatakis A. 2010 - How many bootstrap replicates are necessary? Journal of Computational Biology 17:337-354.

Pegler DN, Rayner RW. 1969 - The Agaric Flora of Kenya. Royal Botanic Gardens, Kew Bulletin 23:347-412.

Reid DA. 1984 - Another British record of Puccinia longicornis. Bulletin of the British Mycological Society 18:27-129.

Ronquist F, Teslenko M, van der Mark P, Ayres DL et al. 2012 - Mr. Bayes 3.2: efficient Bayesian phylogenetic inference and model choice across a large model space. Systematic Biology 61:539-542.

Singer R. 1948 - New and interesting Species of Basidiomycetes. Papers Michigan Academy of Science, Arts and Letters. 32:103-150.

Stamatakis A. 2014 - RAxML version 8: A tool for phylogenetic analysis and post-analysis of large phylogenies. Bioinformatics 30:1312-1313.

Taylor DL, McCormick MK. 2008 - Internal transcribed spacer primers and sequences for improved characterization of basidiomycetous orchid mycorrhizas. New Phytologist 177:1020-1033.

Vellinga EC, Yang ZL. 2003. Volvolepiota and Macrolepiota-Macrolepiota velosa, a new species from China. Mycotaxon 85:183-186.

White TJ, Bruns T, Lee S, Taylor J. 1990 - Amplification and direct sequencing of fungal ribosomal RNA genes for phylogenies. in: M. A. Innis, et al. (Eds.), In: PCR protocols: a guide to methods and applications, Academic Press, San Diego.

Xu J, Sha T, Li YC, Zhao ZW, Yang ZL. 2008 - Recombination and genetic differentiation among natural populations of the ectomycorrhizal mushroom Tricholoma matsutake from southwestern China. Molecular Ecology 17:1238-1247.

Zervakis GI. 2004 - Molecular phylogeny, biogeography and speciation of the mushroom species Pleurotus cystidiosus and allied taxa. Microbiology 150:715-726. 